\title{
Establishment and Application of CIGS Photovoltaic Building Family Library Based on BIM
}

\author{
Xinyu Fan ${ }^{1}$, Wenjun Qin ${ }^{2}$, Chenqi Li ${ }^{1}$, and Feiyu Zhang, ${ }^{2, *}$ \\ ${ }^{1}$ Shenyang Jianzhu University, China \\ ${ }^{2}$ China Energy Group, China
}

\begin{abstract}
With the development of the times, environmental pollution and energy consumption have attracted more and more human attention. The integration of photovoltaic buildings has become an important measure for the sustainable development of buildings.Combining CIGS (copper indium gallium selenide) PV modules with BIM technology for parameter family modeling, applied in BIPV (Building Integrated Photovoltaic) to improve design quality, reduce design errors, acquire and analyze Engineering volume data, and provide technical support for the entire construction process.
\end{abstract}

\section{Introduction of BIM Technology and CIGS Building Integrated Photovoltaic (BIPV)}

BIM is based on state-of-the-art 3D digital design solutions, building a "visualized" digital building model with accurate parameter information on the product technology used, which enables construction engineering to significantly increase efficiency and reduce risk throughout its entire process.CIGS photovoltaic building integration has taken photovoltaic construction a big step forward, but in terms of photovoltaic buildings, more complex technologies are the biggest problem that restricts its promotion and application. Combining BIM technology with CIGS-BIPV can solve this problem better.

\subsection{BIM and parametric modeling}

BIM is the abbreviation of English information for Building Information Modeling. It combines the material information, collection data and physical characteristics of the building to form a comprehensive and complete information base, which can better meet the actual work needs of the participants. The establishment of the BIM building information model is a revolution in the field of architecture and will be a powerful tool for project management. The BIM building information model is applicable to all stages of project construction and can be applied to different areas of the project life cycle.

BIM parametric modeling refers to the establishment and analysis of models by parameters rather than numbers. Simply changing the parameter values in the model can establish and analyze new models; Entities in BIM are presented in the form of components, these components differences are reflected by the adjustment of the parameters, which preserves all the information of the entity as a digital building component.

\subsection{Significance of CIGS-BIPV}

As a fast-growing and efficient thin-film solar cell, CIGS copper-indium gallium selenium thin-film solar cells have attracted much attention both in the international and domestic photovoltaic industry. At present, there are two main types of solar cells for photovoltaic applications. According to the photovoltaic materials used, they are classified into crystalline and thin film types, namely crystalline silicon solar cells and thin-film solar cells. The crystalline materials have monocrystalline silicon, poly-crystalline silicon and so on. Thin film photovoltaic materials include amorphous silicon, copper indium selenium and copper indium gallium selenium, cadmium tellurium and so on. The mono-crystalline silicon material has a high photoelectric conversion rate, but the production cost is large. Although the production cost of poly-crystalline silicon materials is low, the photoelectric conversion efficiency has decreased. However the thin film photovoltaic material copper indium gallium selenium CIGS photovoltaic products have the characteristics of good low light performance, good durability, beautiful form and diverse colors. As a new type of photovoltaic power generation material, CIGS' power generation performance can meet various environmental occasions. And it will become the mainstream technology for the integration of new generation photovoltaic buildings.

BIPV, the integration of photovoltaic buildings, is mainly a way to obtain electrical energy by combining photovoltaic modules with building roofs, walls, sunshades and other components. The building photovoltaic modules generate direct current, which is connected to the inverter through a junction box, and

\footnotetext{
$\overline{\text { * Corresponding author: Zhang_feiu@ } 163 . c o m}$
} 
convert a direct current into an alternating current to meet the power requirements of building loads.

\subsection{Combination of BIM and CIGS-BIPV}

Combining CIGS (copper indium gallium selenium) photovoltaic components with BIM technology, modeling the parameter family, using the created BIM model to improve design quality, reduce design errors, acquire and analyze engineering cost data, and build the entire process for construction provide technical support. Ensure that buildings are up to date, quality, safe, efficient and economical throughout their life cycle. With the large-scale application of CIGS photovoltaic technology in buildings, combined with BIM technology, it will greatly promote the integration of photovoltaic building.

The current family libraries are all provided by special design software. The family libraries of each software are independent and scattered. The model data in the family library is not perfect, and most of them are general information models. Due to the standardized manufacturing of CIGS photovoltaic products, it will form a special family library through BIM, and combine with BIM technology to establish a model of variableparameter photovoltaic component family, which can improve design quality, reduce design errors, reduce engineering cost and optimize architectural design.

\section{The construction of CIGS-BIPV family library based on Revit}

Mr.Dana Smith,president of the American building Smart Alliance,once said that the era of relying on one software to solve all problems is gone.BIM technology does not just refer to the use of a software to work, and Revit is one of the most widely used software in the construction industry BIM system in our country.Each family file in Revit software contains a lot of parameters and information(size,shape,type, and other parameters). Reasonable settings for family parameters make it easy to manage data and modify built models. With the standardized production of CIGS PV modules, accurate, high-quality family library building will play a greater role in CIGS-BIPV.

\subsection{Classification of CIGS photovoltaic components}

Photovoltaic modules as the basic unit of light energy collection, photovoltaic products, size, inclination, installation area, and installation orientation all affect the productivity of the entire system. Photovoltaic building integration mainly has the following several forms of application:

\subsubsection{Photovoltaic roof (including flat roof, inclined roof, etc.)}

There are two types of arrangement of flat roof photovoltaic components: bracket type and embedded type. The arrangement of the inclined roof photovoltaic components is laid-out and embedded.

\subsubsection{Photovoltaic curtain wall}

The photovoltaic curtain walls include exposed-framing curtain walls, clip-on curtain walls, and open-able curtain walls. In addition to the power generation function, the photovoltaic curtain wall must also meet the maintenance functions, use functions, safety performance and aesthetic performance of the building curtain wall.

\subsubsection{Photovoltaic sunshade components}

According to different shade forms, photovoltaic building shades can be divided into three types: photovoltaic horizontal building shading, photovoltaic vertical building shading and photovoltaic baffle building shading. Combining the sunshade device with solar photovoltaic technology can also achieve the harmonious integration of power generation, shading, decoration and other functions. This is the perfect combination of new energy, function and art.

The above three types are the most important forms of photovoltaic building integration applications, in addition, there are photovoltaic railings, photovoltaic grounds and other forms.

\subsection{Establishment of the CIGS-BIPV family library}

In Revit software, the definition of the family category determines how the model is drawn. Due to the classification of photovoltaic components, it is mainly necessary to make various panel families, which will be applied to the nesting family.

\subsubsection{Bracketed photovoltaic flat roof}

As shown in Figure 1. It is necessary to establish curtain wall panels, apply nested families, use the metric generic model family template to establish photovoltaic glass, frame profiles, load into the curtain wall panel family, associate family parameters, add corresponding information, complete the panel family draw. Then use different family templates to establish connectors and brackets. Load them into the project together, and build the model by drawing the roof and beam of the system family. 


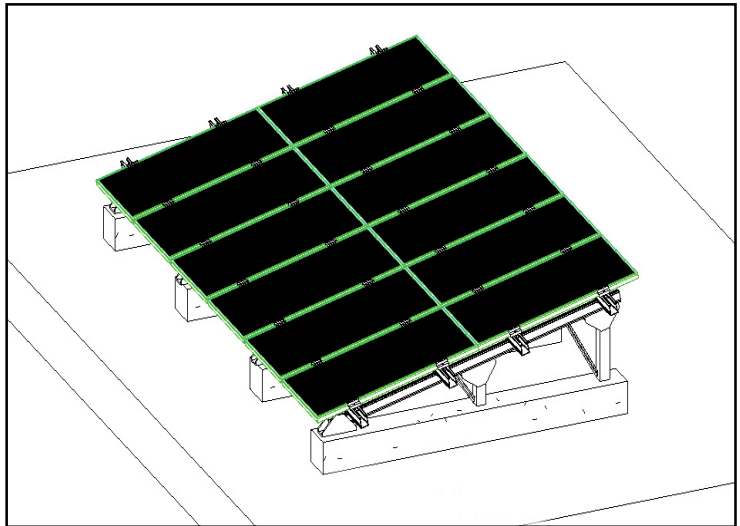

Fig. 1. Demonstration of flat roof photovoltaic component layout

\subsubsection{Embedded photovoltaic inclined roof}

As shown in Figure 2. The same curtain wall panel family can be reused, and the difference from the flat roof is to accurately map the arrangement of the photovoltaic panels of different metal roofs, and then adjust the direction of the connection bracket family.

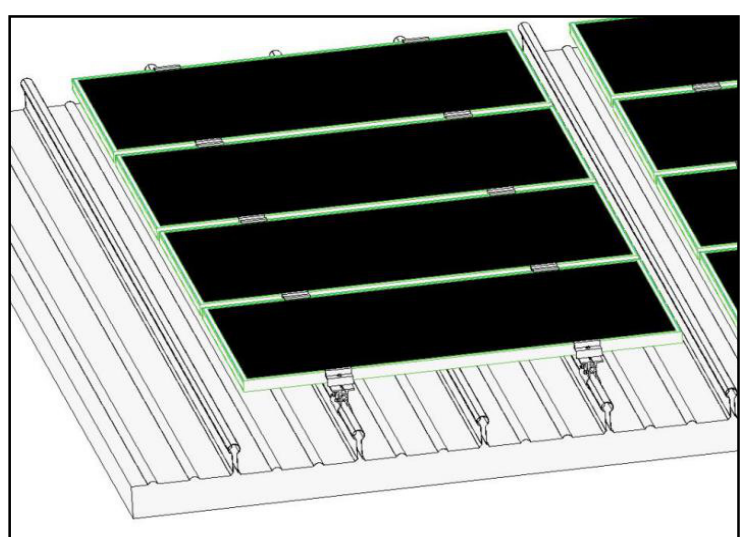

Fig. 2. Demonstration of metal roof photovoltaic component layout

\subsubsection{Exposed-frame photovoltaic curtain wall}

As shown in Figure 3. For the curtain wall, the main family that needs to be built is the curtain wall panel. Unlike the panel family required for the roof, it is also necessary to establish horizontal and vertical keels. The keel styles required for different positions are different, such as the turning position, the connection position with the door and window, the connection position with the opening, the capping position, etc. According to the requirements of different positions, the curtain wall panels with different keels style are established.

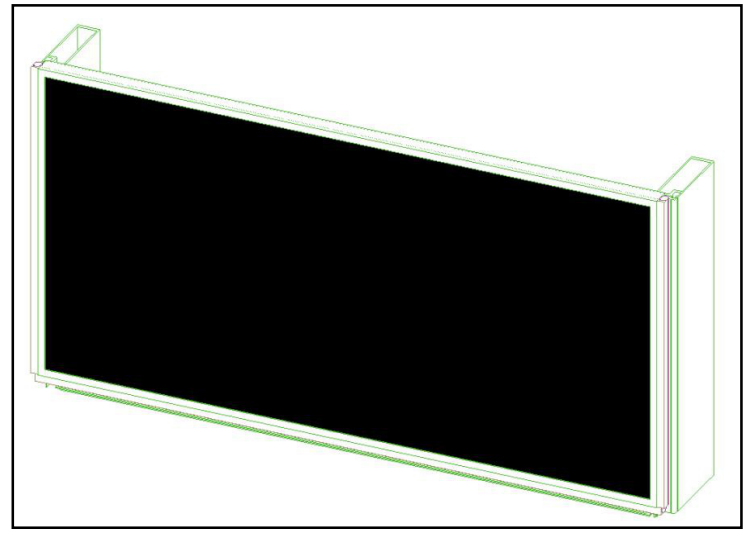

Fig. 3. Demonstration of exposed frame photovoltaic curtain wall panel

\subsubsection{Clip-type photovoltaic curtain wall}

As shown in Figure 4. The clip-type curtain wall will be more generous and beautiful in the whole building. Compared with the frame curtain wall, the drawing of the clip curtain wall panel will be less than the drawing of the frame profile, and the creation of the curtain wall clip will be more.Draw the curtain wall clips to be drawn using the metric generic face-based family template, load it into the curtain wall panel family and adjust the position parameters to load into the project.

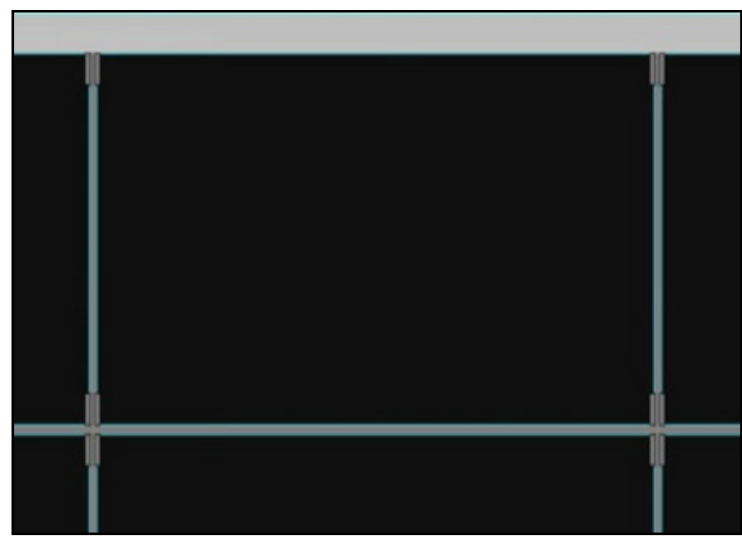

Fig. 4. Demonstration of clip-on photovoltaic curtain wall panels

\subsubsection{Open-able photovoltaic curtain wall}

As shown in Figure 5. The panel family of open-able photovoltaic walls is relatively more complicated to draw. The increase of the opening angle parameter directly affects the difficulty of setting other parameters such as the bracket member. Figure 6 is the parameter setting method. 

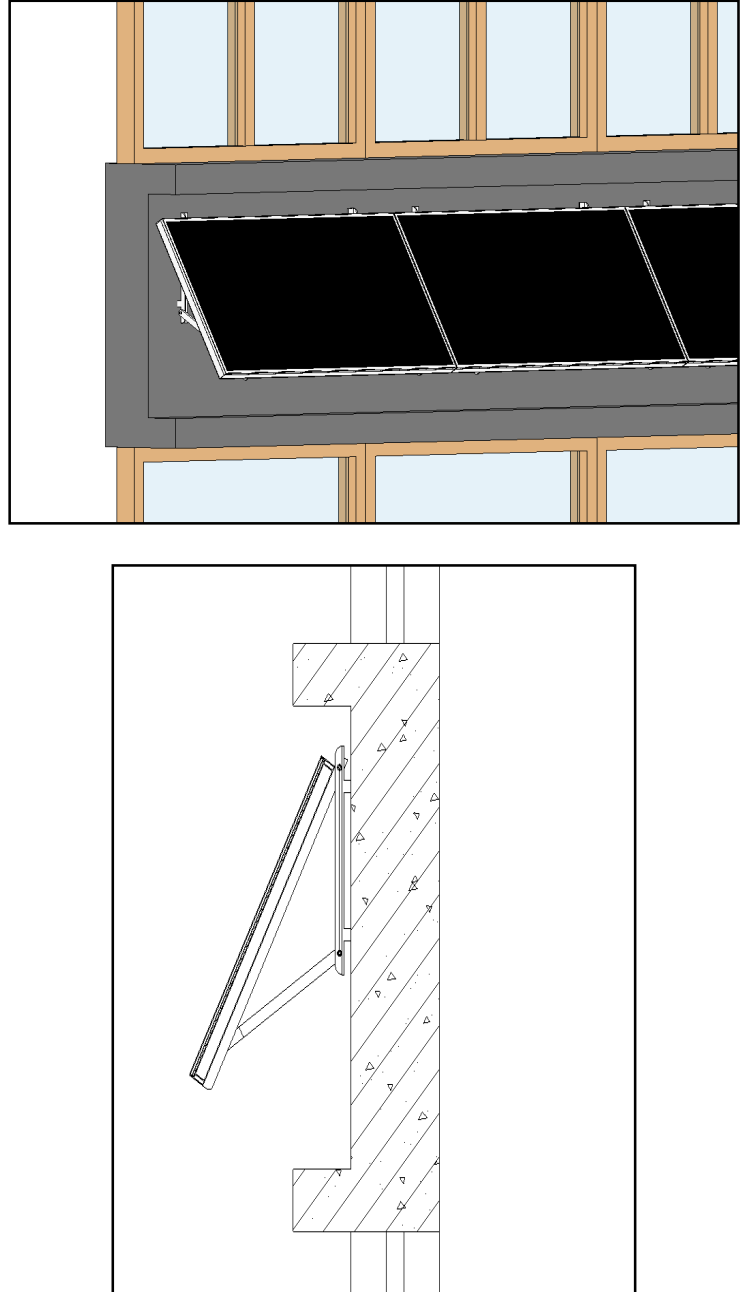

Fig. 5. Open-able photovoltaic curtain wall demonstration

\begin{tabular}{|c|c|c|c|}
\hline Parameter & Value & Formula & Lock \\
\hline \multicolumn{4}{|c|}{ Materials and Finishes } \\
\hline $3 \mathrm{~mm}$ glass material & Glass & ]$=$ & \\
\hline $4 \mathrm{~mm}$ glass material & Photovoltaic glass & $=$ & \\
\hline Profile material & Aluminum & $=$ & \\
\hline Bracket material & Steel & $=$ & \\
\hline \multicolumn{4}{|l|}{ Dimensions } \\
\hline JD & $40.000^{\circ}$ & $=2 * \mathrm{jd}$ & $\Gamma$ \\
\hline$h$ (default) & 900.00 & $=$ & $\Gamma$ \\
\hline h2 (default) & 410.00 & $=\mathrm{h} 1-40 \mathrm{~mm}$ & $\Gamma$ \\
\hline h3 (default) & 897.00 & $=h-3 \mathrm{~mm}$ & $\Gamma$ \\
\hline h4 (default) & 456.00 & $=\mathrm{h} 1+6 \mathrm{~mm}$ & $\Gamma$ \\
\hline jd & $20.000^{\circ}$ & $=$ & $\Gamma$ \\
\hline $\mathrm{I}$ & 1994.00 & $=4 / 3 * \mid 1$ & $\Gamma$ \\
\hline 11 & 1495.50 & $=$ & $\Gamma$ \\
\hline h1 (default) & 450.00 & $=\mathrm{h} / 2$ & $\Gamma$ \\
\hline \multicolumn{4}{|l|}{ Identity Data } \\
\hline
\end{tabular}

Fig. 6. Demonstration of family parameter settings

\subsubsection{Bracket photovoltaic shade}

As shown in Figure 7. The bracket photovoltaic shade is similar to the panel family of the open-able photovoltaic curtain wall. Although the bracket photovoltaic shade usually does not need to adjust the angle, in order to enable the family member to be applied to different projects, it is still necessary to set parameters for the opening angle to realize the angle variation.
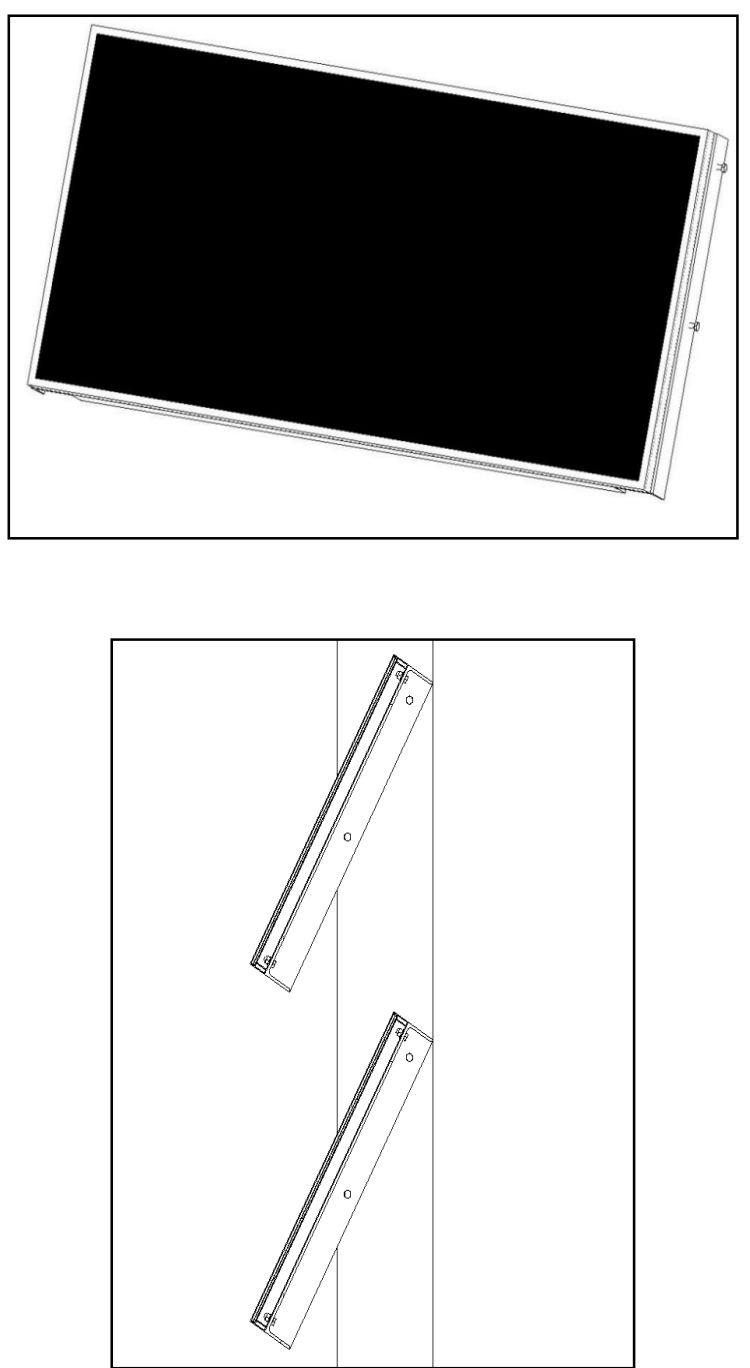

Fig. 7. Bracket photovoltaic shade demonstration

\section{The application of Revit family library in CIGS-BIPV project}

\subsection{Application value of CIGS parametric component family library}

The REVIT software in BIM technology is used to carry out parametric modeling of photovoltaic components, and the data of PV module size, opening angle, connector size and material are set as parameters. The design requirements of PV modules in different buildings are different, and the parameters are adjusted accordingly. Value in conjunction with some applications in the REVIT project, to better complete the architectural optimization design research.Accurate models can also be used to guide construction, or as a model basis for secondary rendering development, greatly improving the accuracy and efficiency of 3D rendering. Parametric modeling of photovoltaic components will strongly promote the construction of photovoltaic standard product component systems in the construction industry, and provide key technical support 
for BIM technology applications in the construction industry.

\subsection{Application process of CIGS family in BIPV project}

Combine one photovoltaic building project to explore the application of CIGS in the project of building photovoltaic integration. The project is a five-story office building, mainly used as a exposing-framed CIGS photovoltaic curtain wall. However,different positions of the building wall, applied to the curtain wall panel style is different.Turning position, connection position with doors and windows, connection position with holes, capping position, etc., according to the needs of different positions, the curtain wall panels with different keel patterns are established.

The curtain wall panels at the ends are adjusted by setting the visibility of the keels and foam bars in the panel family. Different styles of keel components are drawn at the corners and at the junctions with the doors and windows to meet the needs of the project. In the process of drawing, we can find some small errors in the 2D drawings and make timely corrections. Accurate node model building can also be used to guide construction, and the visual features show architectural details more intuitively. At the same time, the model can also be used as the model basis for secondary rendering development. It can be directly imported into Lumion and other software, which greatly improves the accuracy and efficiency of 3D rendering. The creation of the same precise family components is also more convenient for statistics such as engineering quantity and component information, so that the information can be used continuously, and the efficiency of the whole process of the project can be improved significantly.

\section{Conclusion}

In the architectural design research, the PV module parametric variation family matching component information setting can fully play the characteristics of BIM technology such as visualization, optimization, image-ability, parametrization and information completeness. Improve project production efficiency, shorten construction period, reduce construction cost, and promote the development of architectural design field.

As an important part of photovoltaic building, photovoltaic system has a significant impact on the overall performance of the building. We must optimize and control its performance. People's requirements for construction, such as "practical, economical, and aesthetically pleasing", are equally applicable in BIPV design. The introduction of the BIM concept allows architects to more easily deal with the design of various technical and aesthetic issues.

The establishment of the BIM family library allows all components to have a unified specification, which ensures the completeness, availability and transmission of information of the components, which lays a solid foundation for the application of BIM technology in the subsequent stage; based on BIM, we can integrate all kinds of stakeholders of photovoltaic construction industry chain,so that they can get all-round information support and services, and ultimately improve the quality of BIPV design and management; based on BIM, we can better operate the structure of photovoltaic system, lighting function, power generation function, thermal engineering performance, aesthetic performance and other aspects of the content are optimized to ultimately achieve an overall improvement in building quality.

Ministry of Housing and Urbon-Rural Development project name: Application Research of CIGS Solar Thin Film Power Generation Technology in Photovoltaic Building Integration

Project number: 2017-K1-027

\section{References}

1. Seung-Ho Yoo. Solar Energy .1291-1301,(2011)

2. Akash Kumar Shukla, K. Sudhakar, Prashant Baredar. Energy and Buildings ,140, 188-195(2017)

3. Ljubisav Stamenic, Eric Smiley, Karim Karim. Solar Energy ,77, 37-45(2014)

4. Kan He, Ning Gui, Zhifeng Qiu, Bo Pan. Building Energy Efficiency ,44(2016)

5. Jianhua Cheng, Hui Wang. Business Economy ,2931(2012)

6. Hongmei Zhang, Yunhua Yin. Water Resources and Power ,6,193-197(2008)

7. Tao Shi, Jiayan Shen, Jinyang Jiang. New Building Materials ,38-41(2011)

8. Youquan $\mathrm{Xu}$, Yuanyuan Kong. Journal of Engineering Management ,30,28-32(2016)

9. Cui Guo, Quan Liu.Construction Science and Technology ,20-23(2017)

10. Yungui Li, Kuining Qiu. Building Technique Development ,42,3-10(2015) 